$$
\text { Conf- } 9503167--1
$$

UCRL-JC-120862

\title{
PREPRINT
}

\section{EUVE Photometry of SS Cygni: Dwarf Nova Outbursts and Oscillations}

\author{
Christopher W. Mauche
}

This paper was prepared for submittal to the 1995, Proceedings of IAU Colloquium No. 152

Astrophysics in the Extreme Ultraviolet, Cambridge University Press

May 15, 1995

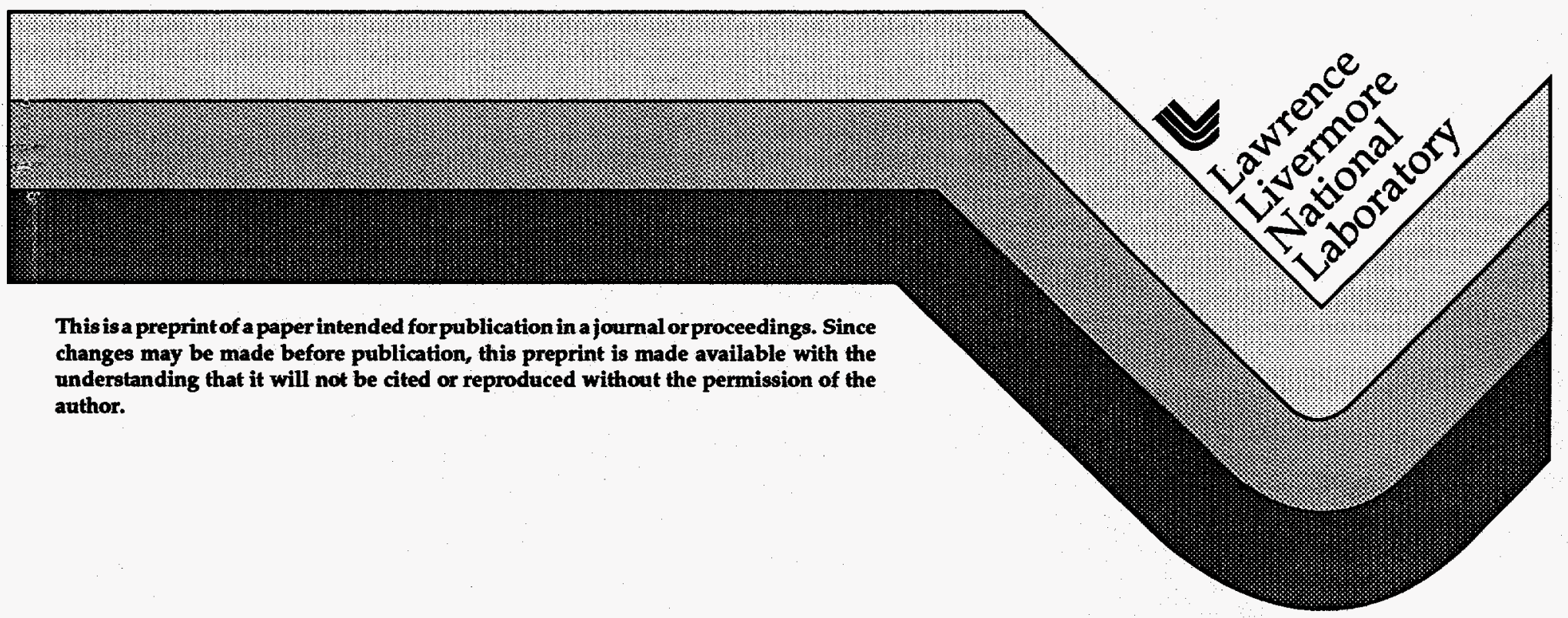




\section{DISCLAIMER}

This report was prepared as an account of work sponsored by an agency of the United States Government. Neither the United States Government nor any agency thereof, nor any of their employees, make any warranty, express or implied, or assumes any legal liability or responsibility for the accuracy, completeness, or usefulness of any information, apparatus, product, or process disclosed, or represents that its use would not infringe privately owned rights. Reference herein to any specific commercial product, process, or service by trade name, trademark, manufacturer, or otherwise does not necessarily constitute or imply its endorsement, recommendation, or favoring by the United States Government or any agency thereof. The views and opinions of authors expressed herein do not necessarily state or reflect those of the United States Government or any agency thereof. 


\section{DISCLAIMER}

Portions of this document may be illegible in electronic image products. Images are produced from the best available original document. 
1995, Proceedings of IAU Colloquium No. 152-Astrophysics in the Extreme Ultraviolet, ed. S. Bowyer \& B. Haisch (Cambridge: Cambridge Univcrsity Press), in press

\title{
EUVE Photometry of SS Cygni: Dwarf Nova Outbursts and Oscillations
}

\author{
By CHRISTOPHER W. MAUCHE \\ Lawrence Livermore National Laboratory, L-41, P.O. Box 808, Livermore, CA 94550, USA
}

We present EUVE Deep Survey photometry and AAVSO optical measurements of the 1993 August and 1994 June/July outbursts of the dwarf nova SS Cygni. The EUV and optical light curves are used to illustrate the different response of the accretion disk to outbursts which begin at the inner edge and propagate outward, and those which begin at the outer edge and propagate inward. Furthermore, we describe the properties of the quasi-coherent $7-9 \mathrm{~s}$ sinusoidal oscillations in the EUV flux detected during the rise and plateau stages of these outbursts.

\section{Introduction}

Simple theory predicts that nonmagnetic cataclysmic variables (CVs; nova-like variables and dwarf novae) should be bright EUV sources. As material sinks through the accretion disk of a CV, half of its specific gravitational potential energy is converted into heat, which is radiated away locally, and half is converted into orbital kinetic energy via the balance of gravitational and centrifugal forces. Consequently, the material in the disk orbiting just above the surface of the white dwarf has a specific kinetic energy equal to $G M_{\mathrm{wd}} / 2 R_{\mathrm{wd}}$, where $M_{\mathrm{wd}}$ and $R_{\mathrm{wd}}$ are the mass and radius of the white dwarf. Unless the white dwarf is rotating near breakup, the material in the disk must dissipate this amount of energy in the boundary layer between the disk and the surface of the white dwarf before accreting onto the star. The luminosity of the boundary layer will be $G M_{\mathrm{wd}} \dot{M} / 2 R_{\mathrm{wd}} \approx 20 \mathrm{~L}_{\odot}$ for a $1 \mathrm{M}_{\odot}$ white dwarf accreting at a rate of $\dot{M}=10^{-8} \mathrm{M}_{\odot} \mathrm{yr}^{-1}$. For the expected size of the boundary layer, this luminosity will be radiated at EUV to soft $\mathrm{X}$-ray energies.

Although the luminosities of the boundary layers in VW Hyi (Mauche et al. 1991) and SS Cyg (Mauche, Raymond, \& Mattei 1995) appear to be lower than predicted by theory by roughly an order of magnitude, these systems are still sufficiently luminous, sufficiently close $(d \lesssim 100 \mathrm{pc})$, and have sufficiently low interstellar column densities $\left(N_{\mathrm{H}} \lesssim 3 \times 10^{19} \mathrm{~cm}^{-2}\right)$ to be observed as bright EUV sources. Indeed, dwarf novae have proven to be particularly rewarding targets for EUVE: their EUV flux varies on time scales ranging from seconds to days, they produce high signal-to-noise ratio spectra, and their spectra are bewilderingly complex. On the down side, dwarf novae can only be observed as targets of opportunity, and so require significant and often urgent effort to observe. The success of the existing EUVE observations of dwarf novae is due to the efforts of the members, staff, and director of the American Association of Variable Star Observers (AAVSO), EUVE Deputy Project Scientist Ron Oliversen, the stafts of the Center for EUV Astrophysics (CEA), the EUVE Science Operations Center at CEA, and the Flight Operations Team at Goddard Space Flight Center.

To date, EUVE has observed the nova-like variable IX Vel (van Teeseling et al. 1995) and the dwarf novae SS Cyg (twice), U Gem (Long et al. 1995), and VW Hyi. Having discussed at length the spectroscopic results from the first EUVE observation of SS Cyg (Mauche, Raymond, \& Mattei 1995), I present here a fuller, though preliminary and largely descriptive, discussion of the photometric results from the Deep Survey (DS) instrument from both EUVE observations of SS Cyg. 
FIGURE 1. DS count rate (filled circles with error bars) and visual magnitude (small dots and histogram) as a function of time from (a) 1993 August and (b) 1994 June/July.

\section{Observations}

EUVE observed SS Cyg in outburst in 1993 August and 1994 June/July. The observations cover the intervals MJD 9216.6 to 9223.1 and MJD 9526.7 to 9536.9 (with a break between MJD 9529.8 and 9532.5). Figure 1 shows the optical light curves of these outbursts. On both occasions, the optical flux was above $V=10$ for $\approx 16$ days, but the 1993 outburst was anomalous in that it took $\approx 5$ days for the light curve to reach maximum, whereas typical outbursts (such as the 1994 outburst) reach maximum in 1 to 2 days. Overplotted on Figure 1 is the log of the count rate of the DS instrument for these outbursts. The DS count rate is seen to increase by a factor of $\approx 330$ for the 1993 outburst, and by a factor of $\approx 1000$ for the 1994 outburst; at the peak of the outburst, the count rate in the DS instrument was $\approx 5$ counts $\mathrm{s}^{-1}$. Figure 1 demonstrates that the EUV light curve of the 1993 outburst rose more quickly than the optical, and that the rise of the EUV light curve of the 1994 outburst was delayed by $\approx 1$ day relative to the optical. It is this delay between the optical and EUV light curves which allows EUVE observations of dwarf novae in outburst to be triggered by "amateur" optical observers. 


\section{Dwarf Nova Outbursts}

The first application of the EUVE photometry of SS Cyg deals with the relative shapes of the optical and EUV light curves shown in Figure 1 and the implications for the processes responsible for dwarf nova outbursts. For an excellent review of the physics of dwarf nova outbursts, see Cannizzo (1993), from which the following synopsis was prepared.

The vertical structure (the run of pressure, temperature, and flux) of an accretion disk is determined by the equations of hydrostatic equilibrium, energy transport by radiation and convection, and, unlike a stellar atmosphere, energy generation via viscous dissipation of orbital shear. Using standard assumptions, it is found that the effective temperature $T_{\text {eff }}$ of the disk as a function of surface density $\Sigma$ is double-valued: in the $\Sigma$ - $T_{\text {eff }}$ plane, the thermal stability of the disk is described by an "S-shaped" curve. That the form of this relationship leads to an instability in the thermal state of the disk can be understood as follows. Imagine that the surface density of a given annulus increases because mass is added to the annulus from "above" (from higher in the gravitational well) faster than it is removed from "below." Initially, the temperature of the annulus will increase monotonically. However, when the surface density reaches some value $\Sigma_{\max }$ when the temperature reaches $\sim 6000 \mathrm{~K}$, hydrogen begins to become ionized, causing a sharp increase in both the opacity and the specific heat of the gas. These microscopic changes in the gas drive macroscopic changes in the vertical structure of the disk as radiation replaces convection as the main source of the transport of energy out of the disk. To cope with these changes, the temperature of the annulus must increase discontinuously to remain in thermal equilibrium. On the upper branch of the S-shaped,curve, the viscous dissipation is higher because the temperature and hence the pressure is higher. Therefore, it is possible for the surface density of the annulus to decrease as mass drains out of the annulus faster than it is added from "above." Initially, the temperature of the annulus will decrease monotonically. However, when surface density reaches some value $\Sigma_{\text {min }}$ when the temperature reaches $\sim 10^{4} \mathrm{~K}$, hydrogen begins to recombine, causing a sharp decrease in both the opacity and the specific heat of the gas. The gas again falls out of thermal equilibrium, this time cooling significantly at fixed surface density.

To describe the response of the full disk to the above instability, it is necessary to specify the dependence of the critical surface densities $\Sigma_{\max }$ and $\Sigma_{\min }$ on radius, and to solve the time-dependent hydrodynamic equations of the conservation of mass, energy, and angular momentum. These calculations demonstrate that when a given annulus reaches the value of $\Sigma_{\max }$ appropriate to its radius and makes the transition to the high state, it generates a heating wave which transforms the entire disk to the high state. This disturbance can begin in the inner disk and sweep outward, or begin in the outer disk and sweep inward, generating, respectively, inside-out and outside-in outbursts. Since mass is predominantly transferred inward through the disk by viscous dissipation, the minimum surface density $\Sigma_{\min }$ is reached first in the outer disk. When that annulus makes the transition to the low state, it generates a cooling wave which transforms the entire disk to the low state. The cooling wave shuts down the disk by moving gas to larger radii, raising it out of the gravitational well and transforming its thermal energy into gravitational potential energy.

The differences between the inside-out and outside-in outbursts qualitatively describe the differences in the optical and EUV light curves shown in Figure 1. The 1993 outburst was of the inside-out variety. As the heating wave swept outward though the disk, it transformed successively larger annuli to the high state, and in the process set this material in motion toward the white dwarf, constantly increasing the rate at which 
the material would reach the inner disk and boundary layer and there radiate at EUV wavelengths. The transition to the high state was relatively slow, because the heating wave had to "swim upstream" against the surface density profile of the disk and the inward-spiraling gas. The 1994 outburst, on the other hand, was of the outside-in variety. As the heating wave swept inward through the disk, it transformed successively smaller annuli to the high state, and in the process set this material in motion toward the white dwarf. News of the outburst arrived at the inner disk and boundary layer with the arrival of the heating wave, just ahead of the flow of material which would power the boundary layer. The transition to the high state was relatively fast, because the heating wave "ran down" the surface density profile of the disk, in the same direction as the inward-spiraling gas. The delay of $\approx 1$ day between the rise of the optical and EUV light curves is the length of time required for the heating wave to travel the length of the accretion disk.

This delay of $\approx 1$ day between the EUV and optical light curves is longer than the delay of $\approx 0.5-0.75$ day between the FUV $(950-1150 \AA)$ and optical light curves measured by Cannizzo, Wheeler, \& Polidan (1986) for the 1980 May outside-in outburst of SS Cyg. The length of the FUV delay is shorter than the EUV delay because the disk itself produces the FUV flux and the heating wave travels a shorter distance before heating an annulus of the disk to the temperature $(\sim 30,000 \mathrm{~K})$ required for it to radiate in the FUV. This difference between the FUV and EUV light curves of SS Cyg highlights the importance of the above EUV light curves: the optical through FUV light curves measure the response of the disk to the outburst; the EUV light curves measure the rate at which material arrives at the boundary layer. The combination of the EUV and optical, UV, and/or FUV light curves makes for a powerful diagnostic of the mechanisms responsible for dwarf nova outbursts. To follow up these results, we plan to observe VWW Hyi this summer simultaneously with Voyager and EUVE.

\section{Dwarf Nova Oscillations}

Superposed on the long-term photometric variations associated with its dwarf nova outbursts, SS Cyg exhibits quasi-coherent photometric oscillations ("dwarf nova oscillations," see, e.g., Patterson 1981; Warner 1995) on a time scale measured in seconds. Optical oscillations were detected by Patterson, Robinson, \& Kiplinger (1978) with a period of $9.74 \mathrm{~s}$, by Horne \& Gomer (1980) with periods of 8.23 and $8.50 \mathrm{~s}$, and by Patterson (1981) with periods of $10.72,10.90$, and $8.9 \mathrm{~s}$. Hildebrand, Spillar, \& Stiening (1981) tracked the oscillation over an interval of 6 days and observed its period fall from $7.53 \mathrm{~s}$ to $7.29 \mathrm{~s}$ and then rise again to $8.54 \mathrm{~s}$. At soft X-ray energies, Córdova et al. (1980) and Córdova et al. (1984) detected oscillations in HEAO 1 A-2 data at a period of $9 \mathrm{~s}$ and $11 \mathrm{~s}$, respectively. Jones \& Watson (1992) detected oscillations in EXOSAT LE data at periods between 7.4 and $10.4 \mathrm{~s}$.

To search for oscillations in the EUV flux measured by the DS instrument, we proceed as follows. First, for each of the $i$ satellite orbits, we construct DS count rate light curves with $1 \mathrm{~s}$ time resolution. Next, we phase-fold these light curves using a range of trial periods $P_{j}=P_{1}+\left[j\left(P_{2}-P_{1}\right) / j_{\max }\right]$ for $j=0,1, \ldots, j_{\max }$. Finally, we test for an oscillation with the given period during the given orbit by means of the $\chi^{2}$ statistic: $\chi_{i, j}^{2}=$ $\sum_{k=1}^{k_{\max }}\left(r_{k}-\langle r\rangle\right)^{2} / \sigma_{k}^{2}$, where $r_{k}$ is the count rate in phase bin $k,\langle r\rangle=\sum_{k=1}^{k_{\max }} r_{k} / k_{\max }$, and $\sigma_{k}=\sqrt{r_{k}}$. For an oscillation to be detected by this means, it must have a period within the range of trial periods, it must have a significant amplitude, and it must be coherent in phase and period over the $\$ 2000$ s interval during each orbit when the source is visible to the satellite. 
C. W. Mauche: EUVE Photometry of SS Cygni

FIGURE 2. DS count rate (filled circles with error bars) and pulsation period (diamonds) as a function of time from (a) 1993 August and (b) 1994 June/July. The size of the diamonds correspond to the relative size of the $\chi^{2}$ statistic (see text).

To present the results of these calculations, we show in Figure 2 the $\chi^{2}$ statistic in the form of a pseudo-contour diagram along with the log of the DS count rate. It is apparent from this figure that coherent oscillations exist in the DS count rate for both outbursts. For the 1993 outburst, midom jwiks in the $\chi^{2}$ surface are present very early in the outburst, but the oscillation does not stabilize until $\approx$ MJD 9219 when the count rate is $\approx 2.8$ counts $\mathrm{s}^{-1}$. The period of the oscillation is initially $\approx 8.6 \mathrm{~s}$, falls over the next day or so to $\approx 7.8 \mathrm{~s}$, and then asymptotically approaches $\approx 7.5 \mathrm{~s}$ over the next few days. For the 1994 outburst, the oscillation turns on during the fast rise to outburst on $\approx$ MJD 9528 when the count rate is $\approx 2.3$ counts $\mathrm{s}^{-1}$. The period of the oscillation is initially $\approx 8.9 \mathrm{~s}$, falls to $\approx 7.2 \mathrm{~s}$ (the shortest period ever observed in SS Cyg, or in any other dwarf nova) within a day, and rebounds to $\approx 7.4 \mathrm{~s}$ by the end of the observation on MJD 9529.8. When observations resume $\approx 3$ days later, the period of the oscillation is $\approx 7.6 \mathrm{~s}$ and rises slowly over the next few days to $\approx 8.0 \mathrm{~s}$.

Other quantitative aspects of the oscillations are as follows. First, as is evident from Figure 2, the period of the oscillation correlates with the count rate, being long when the count rate is low and short when the count rate is high. Furthermore, it appears from the 1994 outburst that at a given count rate the period on the rise to outburst is to first 
FIGURE 3. Power spectrum of the DS count rate from the half-hour interval centered on MJD 9222.06. The fundamental lies at $7.50 \mathrm{~s}$ and the positions of the first and second harmonics and the first subharmonic are indicated.

order equal to the period on the decline. This is true despite the fact that the period derivative $\dot{P}$ is significantly higher on the rise compared to that on decline. Evidently, the period of the oscillation does not depend to any significant degree on $\dot{P}$, but only on the DS count rate, and, by inference, only on the mass-accretion rate through the boundary layer. Second, the amplitude of the oscillation correlates with the count rate, being high $(\approx 100 \%)$ when the count rate is low, and moderate $(20 \% \pm 10 \%)$ when the count rate is high. Furthermore, the amplitude of the oscillation is equal at a given count rate between the 1993 and 1994 outbursts. This fact is particularly striking in the interval $\log$ DS count rate $=-1.5$ to 0 , which is traversed slowly on the rise of the 1993 outburst, and very rapidly on the rise of the 1994 outburst. Finally, based on the shape of the phase-folded light curves and on the absence of power at any of the harmonics in power spectra of the light curves (see Fig. 3), the oscillations are purely sinusoidal.

What is the physical mechanism responsible for these ascillations? For a review of this subject, refer to Warner (1995). The low period stability of the oscillations rules out the rotating white dwarf (the DQ Her mechanism) as well as non-radial pulsations of the white dwarf as the cause of the oscillations; pulsations are observed in high inclination systems, ruling out the eclipse by the white dwarf of luminous blobs of material in the inner disk and boundary layer; $r$-modes and trapped g-modes fail because more than one mode would be excited; oscillations of the accretion disk fail because they are not confined to a particular annulus and hence to a particular period. Viable mechanisms are more difficult to construct. King (1985) describes a mechanism employing transient magnetic fields which channel electrons from a hot corona down to the surface of the white dwarf. Molteni, Sponholz, \& Chakrabarti (1995) argue that shocks are an inevitable consequence of gas flow near the inner edge of an accretion disk and that shock oscillations lead to a cycling of the accretion luminosity when the cooling time of the shocked gas is comparable to the radial infall time. Warner \& Livio (1995) propose that the oscillations are due to the combined action of the differentially rotating surface layers of the white dwarf and magnetically controlled accretion.

The EUVE observations of the 1994 outburst of SS Cyg severely constrain the Warner $\&$ Livio model. The time derivative of the kinetic energy of the rotating surface layers of the white dwarf is $\dot{E}_{\mathrm{K}}=4 \pi^{2} I \dot{P} / P^{3}$ where $I=M R^{2}$ is the moment of inertia of 
the surface layers (modeled as a thin-walled hollow cylinder of mass $M$ and radius $R$ ). During the rise of the 1994 outburst, $\dot{P} \approx 2 \mathrm{~s} / 0.5$ day $\approx 5 \times 10^{-5} \mathrm{~s} \mathrm{~s}^{-1}$ and $P \approx 8 \mathrm{~s}$, hence $\dot{E}_{\mathrm{K}} \approx 2 \times 10^{35}\left(M / 10^{-10} \mathrm{M}_{\odot}\right)\left(\mathrm{R} / 5.5 \times 10^{8} \mathrm{~cm}\right)^{2} \mathrm{erg} \mathrm{s}^{-1}$. This value is $\gtrsim 100$ times the measured peak boundary layer luminosity (Mauche, Raymond, \& Mattei 1995), implying, at a minimum, that the mass of the rotating surface layers must be significantly less than $10^{-12} \mathrm{M}_{\odot}$.

The author is pleased to acknowledge the invaluable contributions to this research by the members, staff, and director, J. Mattei, of the AAVSO. The author greatly benefited by conversations with J. Cannizzo and B. Warner concerning the dwarf nova outburst mechanism and dwarf nova oscillations. B. Warner, M. Livio, and S. Chakrabarti generously provided copies of material prior to publication. This work was performed under the auspices of the U.S. Department of Energy by Lawrence Livermore National Laboratory under contract No. W-7405-Eng-48.

\section{REFERENCES}

Cannizzo, J. K. 1993 The Limit Cycle Instability in Dwarf Nova Accretion Disks. In-Accretion Disks in Stellar Systems (ed. J. C. Wheeler). pp. 6-40. World Scientific.

Cannizzo; J. K., Wheeler, J. C., \& Polidan, R. S. 1986 Dwarf Nova Burst Asymmetry and the Physics of Accretion Disks. Ap.J. 301, 634-640.

Córdova, F. A., Chester, T. J., Mason, K. O., Kahn, S. M., \& Garmine, G. P. 1984 Observations of Quasi-Coherent Soft X-ray Oscillations in U Geminorum and SS Cygni. Ap.J. 278, 739-753.

Cóndova, F. A., Chester, T. J., Tuohy, I. R., \& Garmine; G. P. 1980 Soft X-ray Pulsations from SS Cygni. Ap.J. 235, 163-176.

Hildebrand, R. H., Spillar, E. J., \& Striening, R. F. 1981 Observations of Fast Oscillations in SS Cygni. Ap.J. 243, 223-227.

HoRne, K., \& GOMER, R. 1980 Phase Variability in the Rapid Optical Oscillations of SS Cygni. Ap.J. 237, 845-849.

JoNES, M. H., \& WATSON, M. G. 1992 The EXOSAT Observations of SS Cygni. M.N.R.A.S. 257, 633-649.

KING, A. R. 1985 The Origin of Soft X-ray Pulsations in Dwarf Novae at Outburst and the DQ Herculis Phenomenon. Nature 313, 291-292.

Long, K. S., Mauche, C. W., Szkody, P., \& Mattei, J. A. 1995 EUVE Observations of U Gem. In Proceedings of the Padova-Abano Conference on Cataclysmic Variables in press.

Mauche, C. W., Raymond, J. C., \& Mattei, J. A. 1995 EUVE Observations of the Anomalous 1993 August Outburst of SS Cygni. Ap.J. 446, in press.

Mauche, C. W. WAde, R. A., Polidan, R. S., van der Woerd, H., \& Paerels, F. B. S. 1991 On the X-ray Emitting Boundary Layer of the Dwarf Nova VW Hydri. Ap.J. 372, 659-663.

Molteni, D.; Sponholz; H.; \& Chakrabarti, S. K. 1995 Resonance Oscillations of the Radiative Shocks in Accretion Disks around Compact Objects. Ap.J. submitted.

Patterson, J. 1981 Rapid Oscillations in Cataclysmic Variables. VI. Periodicities in Erupting Dwarf Novae. Ap.J.S. 45, 517-539.

Patterson, J., Robinson: E. L., \& Kiplinger, A. L. 1986 Detection of a 9.735 Second Periodicity in the Light Curve of SS Cygni. Ap.J. 226, L137-L139.

WARNER, B. 1995 Cataclysmic Variable Stars. Cambridge.

Warner: B., \& Lrvio, M. 1995 A Model for Dwarf Nova Oscillations. Ap. J. submitted.

van Teeseling: A., Drake, J. J., Drew, J. E., Hoare, M. G., \& Verbunt, F. 1995 Rosat and EUVE Observations of the Nova-like Variable IX Velorum. Astr. Ap. submitted. 

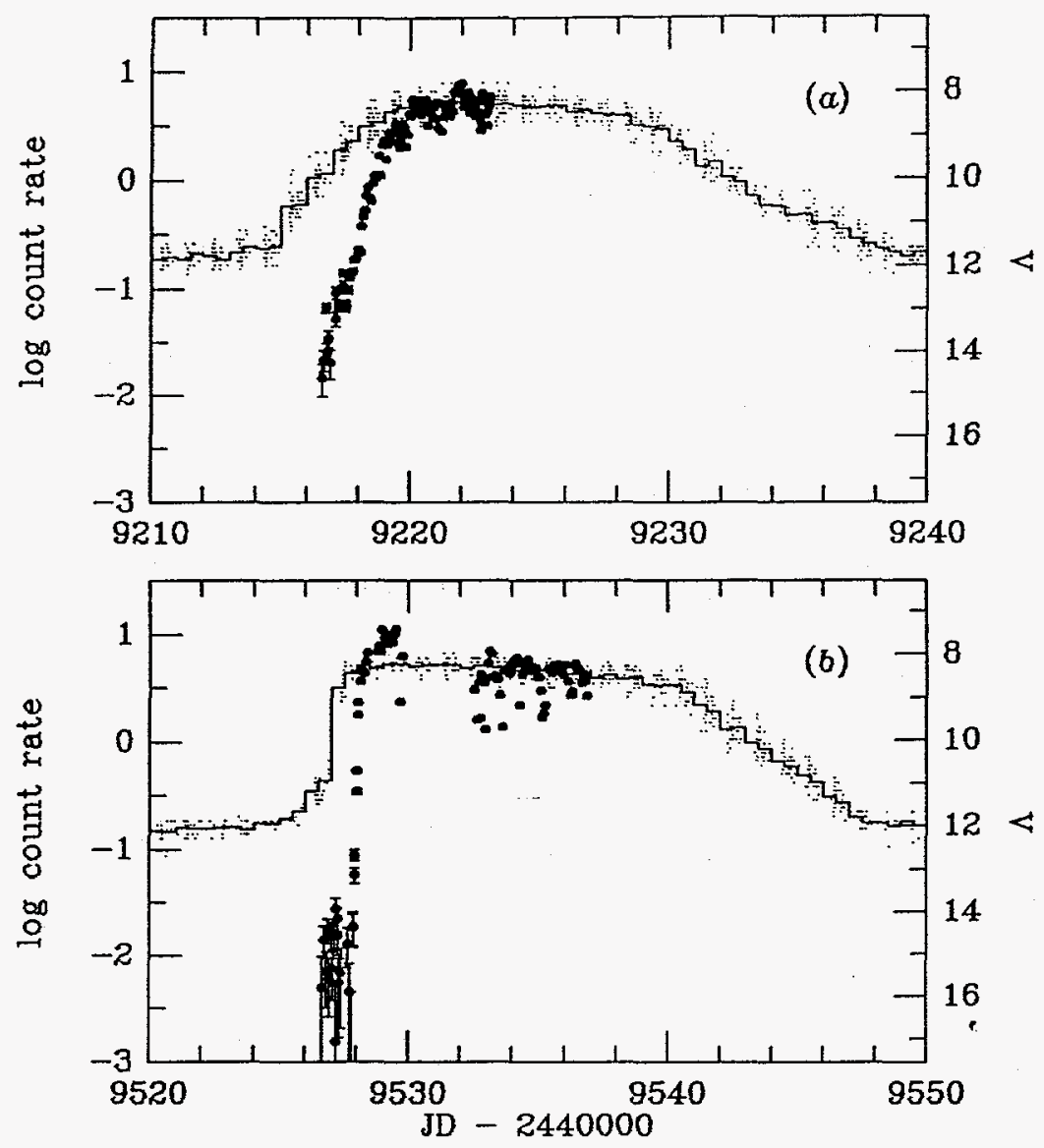

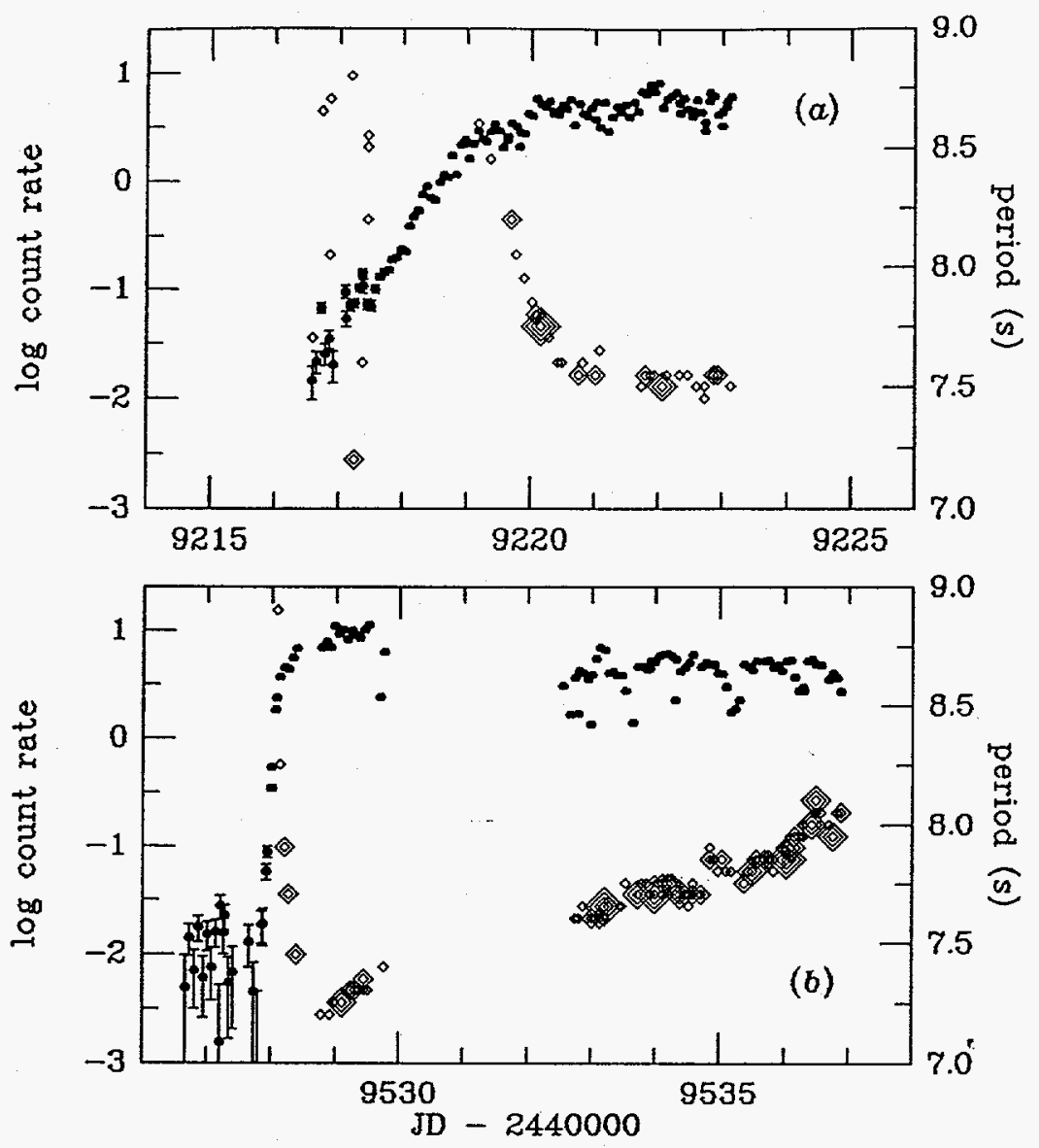


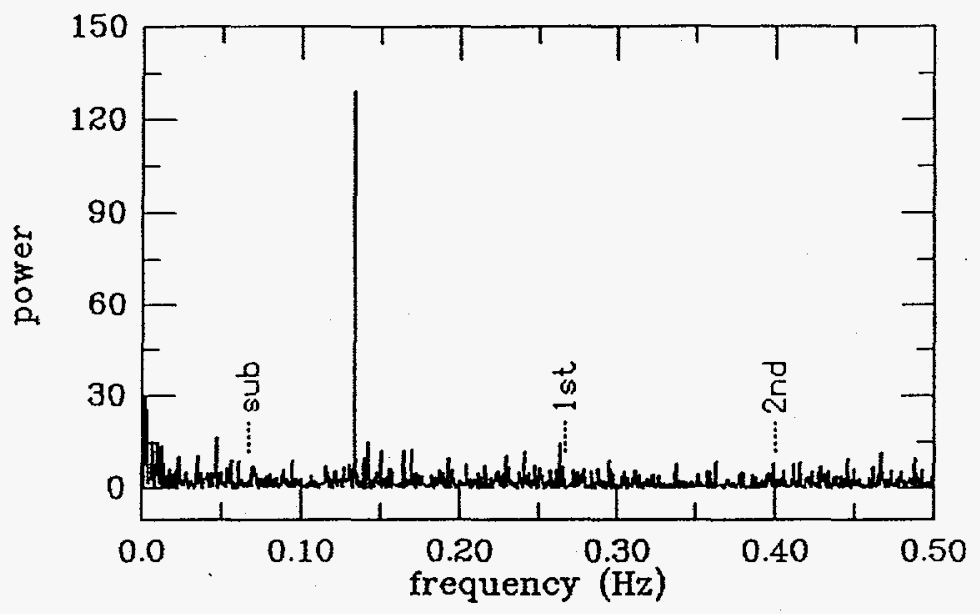

